\title{
Analysis of Exponentially Tapered Piezoelectric Bimorph Actuator
}

\author{
Jungsoon Kim (1), Moojoon Kim (2) and Kanglyeol Ha (2)
}

(1) Media Engineering, Tongmyong University, Busan, Korea

(2) Physics, Pykyong National University, Busan, Korea

PACS: $43.38 . \mathrm{Fx}, 43.58 . \mathrm{Bh}$

\section{ABSTRACT}

In order to investigate the most effective shape for a piezoelectric bimorph actuator, equations giving the free input admittance for an exponentially tapered vibrator were derived. Five piezoelectric bimorph actuators with different shapes were fabricated and their characteristics were analyzed using the derived equations and by measurements. The experimental and theoretical results were in good agreement, and they suggested that the resonant frequency and displacement can be controlled by the tapered shape.

\section{INTRODUCTION}

The piezoelectric bimorph actuator has been used in a wide range of application fields as a sensor, a vibration source, and a position controller[1,2,3]. The shape of the bimorph has been changed to improve its characteristics, especially, in the case of cantilever-type bimorphs[4]. The tip of an atomic force microscope(AFM) is a good example of a cantilever bimorph[5]. However, the analysis of the characteristics of actuators is mainly performed using numerical methods because it is difficult to solve the wave equation including the shape factor analytically[6,7]. Therefore, the optimum design of the bimorph is not easy to determine because of the enormous number of calculations. In this study, to investigate the most effective shape for a piezoelectric bimorph actuator, which can improve the frequency bandwidth, equations to calculate the free input admittance of an exponentially tapered actuator are derived. Five piezoelectric bimorph actuators with different shapes are fabricated and their characteristics are examined through the derived equations and by measurements.

\section{ANALYSIS OF A SIMPLIFIED MODEL}

The structure of the exponentially tapered bimorph actuator is shown in Figure 1(a) and Figure 1(b). An elastic plate with thickness $2 t_{m}$ is inserted between two piezoelectric plates. The poling direction of the two piezoelectric plates is parallel with the $z$-axis. The width of the actuator $(x)$ decreases exponentially from the fixed left end (width $b$ ) to the freely vibrating right end (width $a$ ). We assumed that the variation of the width is given by

$$
x=b \exp \left[\frac{y}{l} \ln \left(\frac{a}{b}\right)\right] .
$$

with $b>a$. If the length $l$ is sufficiently large to be comparable to $b$ and the thickness $t_{p}$, the constitutive equation is written as follows[8]:

$$
\begin{gathered}
S_{2}=s_{22}^{E} T_{2}+d_{32} E_{3} \\
D_{3}=d_{32} T_{2}+\varepsilon_{33}^{T} E_{3},
\end{gathered}
$$

where $S_{2}$ is the strain, $s_{22}^{E}$ is the elastic compliance under a constant electric field, $T_{2}$ is the stress, $d_{32}$ is the dielectric constant, $E_{3}$ is the electric field, $D_{3}$ is the electric charge density, and $\varepsilon_{33}^{T}$ is the permittivity under constant stress. Including the
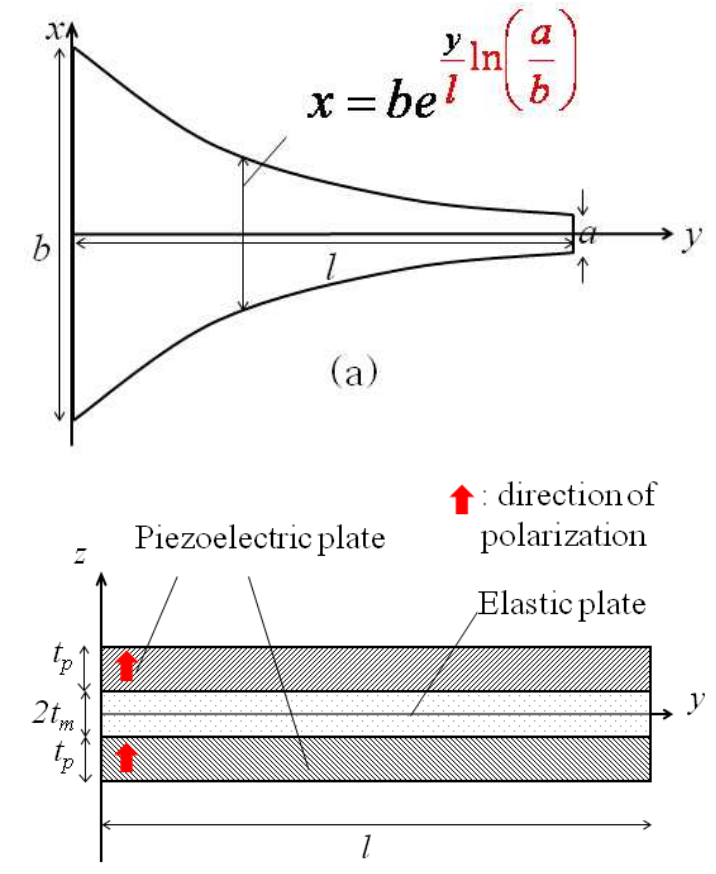

Figure 1: Calculation model for bimorph actuator.

tapered shape given by eq. (1) in the analysis of the bending mode vibration[8], the wave equation is given as[9]

$$
\frac{\partial^{4} \xi_{p}}{\partial y^{4}}+2 \alpha \frac{\partial^{3} \xi_{p}}{\partial y^{3}}+\alpha^{2} \frac{\partial^{2} \xi_{p}}{\partial y^{2}}-k_{p}^{2} \xi_{p}-Q_{V} \alpha^{2}=0
$$

where

$$
\begin{aligned}
& k_{p}=\sqrt{\frac{2 \omega^{2}\left(t_{p} \rho_{p}+t_{m} \rho_{m}\right)}{K_{M}}}, \quad Q_{V}=\frac{N V}{K_{M}}, \\
& \alpha=\frac{1}{l} \ln \left(\frac{a}{b}\right), \quad N=\left(t_{p}+2 t_{m}\right) \frac{d_{32}}{s_{22}^{E}},
\end{aligned}
$$




$$
K_{M}=\frac{2 Y_{M} t_{m}^{3}}{3}+\frac{2 t_{p}\left(t_{p}^{2}+3 t_{p} t_{m}+3 t_{m}^{2}\right)}{3 s_{22}^{E}\left(1-k_{32}^{2}\right)}-\frac{t_{p} s_{22}^{E} N^{2} k_{32}^{2}}{2 d_{32}^{2}\left(1-k_{32}^{2}\right)},
$$

$t_{p}$ is the thickness of the piezoelectric plate, and $\rho_{m}$ and $\rho_{p}$ are the densities of the piezoelectric plate and the elastic material, respectively. $V$ is the voltage applied to the bimorph actuator and $Y_{M}$ is the Young's modulus of the elastic material. To obtain the displacement $\xi_{p}$, that is, the solution of eq. (3), the mechanical boundary conditions given by eqs. (4) and (5) are applied to eq. (3).

$$
\begin{aligned}
& \xi_{p}=0 \& \frac{\partial \xi_{p}}{\partial y}=0, \quad \text { at } \quad y=0 \\
& M_{p}=0 \& F_{p}=0, \quad \text { at } \quad y=l
\end{aligned}
$$

After some manipulation, the displacement of the actuator can be obtained as

$$
\xi_{p}=\left[\begin{array}{c}
\frac{M_{c 1}}{\Lambda_{c}} e^{-h_{1} \frac{y}{2}}+\frac{M_{c 2}}{\Lambda_{c}} e^{-h_{2} \frac{y}{2}}+\frac{M_{c 3}}{\Lambda_{c}} e^{-h_{3} \frac{y}{2}} \\
+\frac{M_{c 4}}{\Lambda_{c}} e^{-h_{4} \frac{y}{2}}-\frac{\alpha^{2} N}{k_{p}^{4} K_{M}}
\end{array}\right] V,
$$

where

$$
\begin{aligned}
& h_{1}=\alpha+\sqrt{\alpha^{2}-4 k_{p}^{2}}, \quad h_{2}=\alpha-\sqrt{\alpha^{2}-4 k_{p}^{2}}, \\
& h_{3}=\alpha+\sqrt{\alpha^{2}+4 k_{p}^{2}}, \quad h_{4}=\alpha-\sqrt{\alpha^{2}+4 k_{p}^{2}}, \\
& \Lambda_{c}=e^{\frac{l}{2}\left(h_{1}+h_{2}\right)}\left(h_{3}-h_{4}\right)\left(h_{1}-h_{2}\right) h_{3}^{2} h_{4}^{2}+e^{\frac{l}{2}\left(h_{1}+h_{3}\right)}\left(h_{3}-h_{1}\right)^{2} h_{2}^{2} h_{4}^{2} \\
& -e^{\frac{l}{2}\left(h_{1}+h_{4}\right)}\left(h_{4}-h_{1}\right)^{2} h_{2}^{2} h_{3}^{2}-e^{\frac{l}{2}\left(h_{2}+h_{3}\right)}\left(h_{3}-h_{2}\right)^{2} h_{4}^{2} h_{1}^{2} \\
& +e^{\frac{l}{2}\left(h_{2}+h_{4}\right)}\left(h_{4}-h_{2}\right)^{2} h_{1}^{2} h_{3}^{2}+e^{\frac{l}{2}\left(h_{3}+h_{4}\right)}\left(h_{3}-h_{4}\right)\left(h_{1}-h_{2}\right) h_{1}^{2} h_{2}^{2}, \\
& A=e^{\frac{l}{2} h_{2}}\left(h_{4}-1\right) h_{2} h_{3}^{2} h_{4}^{2}+e^{\frac{l}{2} h_{3}}\left(h_{3}-h_{1}\right) h_{3} h_{2}^{2} h_{4}^{2} \\
& +e^{\frac{l}{2} h_{4}}\left(h_{1}-h_{4}\right) h_{4} h_{2}^{2} h_{3}^{2} \text {, } \\
& B=e^{\frac{l}{2}\left(h_{2}+h_{3}\right)}\left(h_{3}-2 \alpha\right)\left(h_{2}-h_{3}\right) h_{4}^{2}-e^{\frac{l}{2}\left(h_{2}+h_{4}\right)}\left(h_{4}-2 \alpha\right) \\
& \times\left(h_{2}-h_{4}\right) h_{3}^{2}+e^{\frac{l}{2}\left(h_{3}+h_{4}\right)}\left(h_{1}-2 \alpha\right)\left(h_{3}-h_{4}\right) h_{2}^{2}, \\
& C=e^{\frac{l}{2} h_{1}}\left(h_{4}-1\right) h_{1} h_{3}^{2} h_{4}^{2}+e^{\frac{l}{2} h_{3}}\left(h_{3}-h_{2}\right) h_{3} h_{1}^{2} h_{4}^{2} \\
& +e^{\frac{l}{2} h_{4}}\left(h_{2}-h_{4}\right) h_{4} h_{1}^{2} h_{3}^{2} \text {, } \\
& D=e^{\frac{l}{2}\left(h_{1}+h_{3}\right)}\left(h_{3}-2 \alpha\right)\left(h_{1}-h_{3}\right) h_{4}^{2}-e^{\frac{l}{2}\left(h_{1}+h_{4}\right)}\left(h_{4}-2 \alpha\right) \\
& \times\left(h_{1}-h_{4}\right) h_{3}^{2}+e^{\frac{l}{2}\left(h_{3}+h_{4}\right)}\left(h_{2}-2 \alpha\right)\left(h_{3}-h_{4}\right) h_{1}^{2}, \\
& E=e^{\frac{l}{2} h_{1}}\left(h_{3}-h_{1}\right) h_{1} h_{2}^{2} h_{4}^{2}+e^{\frac{l}{2} h_{2}}\left(h_{2}-h_{3}\right) h_{2} h_{1}^{2} h_{4}^{2} \\
& +e^{\frac{l}{2} h_{4}}\left(h_{1}-h_{2}\right) h_{1}^{2} h_{2}^{2} h_{4} \text {, } \\
& F=e^{\frac{l}{2}\left(h_{1}+h_{2}\right)}\left(h_{3}-2 \alpha\right)\left(h_{2}-h_{1}\right) h_{4}^{2}-e^{\frac{l}{2}\left(h_{1}+h_{4}\right)}\left(h_{1}-2 \alpha\right) \\
& \times\left(h_{4}-h_{1}\right) h_{2}^{2}+e^{\frac{l}{2}\left(h_{2}+h_{4}\right)}\left(h_{2}-2 \alpha\right)\left(h_{4}-h_{2}\right) h_{1}^{2}, \\
& G=e^{\frac{l}{2} h_{1}}\left(h_{4}-h_{1}\right) h_{1} h_{2}^{2} h_{3}^{2}+e^{\frac{l}{2} h_{2}}\left(h_{2}-h_{4}\right) h_{2} h_{3}^{2} h_{1}^{2} \\
& +e^{\frac{l}{2} h_{3}}\left(h_{1}-h_{2}\right) h_{1}^{2} h_{2}^{2} h_{3} \text {, } \\
& H=e^{\frac{l}{2}\left(h_{1}+h_{2}\right)}\left(h_{4}-2 \alpha\right)\left(h_{2}-h_{1}\right) h_{3}^{2}-e^{\frac{l}{2}\left(h_{1}+h_{3}\right)}\left(h_{1}-2 \alpha\right) \\
& \times\left(h_{3}-h_{1}\right) h_{2}^{2}+e^{\frac{l}{2}\left(h_{2}+h_{3}\right)}\left(h_{2}-2 \alpha\right)\left(h_{3}-h_{2}\right) h_{1}^{2}, \\
& \begin{array}{l}
M_{c 1}=e^{\frac{l h_{1}}{2}}\left\{\alpha^{2} A+4 B k_{p}^{4}\right\} \frac{N}{K_{M}}, \\
M_{c 2}=-e^{\frac{l h_{2}}{2}}\left\{\alpha^{2} C+4 D k_{p}^{4}\right\} \frac{N}{K_{M}}, \\
M_{c 3}=-e^{\frac{l h_{3}}{2}}\left\{\alpha^{2} E+4 F k_{p}^{4}\right\} \frac{N}{K_{M}}, \\
M_{c 4}=e^{\frac{l h_{4}}{2}}\left\{\alpha^{2} G+4 H k_{p}^{4}\right\} \frac{N}{K_{M}} .
\end{array}
\end{aligned}
$$

From the relationship between the electric current and the voltage, the input free admittance of the actuator is given by

$$
\begin{aligned}
& Y=j \omega b \frac{N}{2 \Lambda_{c}} \\
& \times\left\{\begin{array}{c}
\frac{h_{1}^{2}}{h_{2}} M_{c 1}\left(e^{h_{2} \frac{y}{2}}-1\right)+\frac{h_{2}^{2}}{h_{1}} M_{c 2}\left(e^{h_{1} \frac{y}{2}}-1\right) \\
+\frac{h_{3}^{2}}{h_{4}} M_{c 3}\left(e^{h_{4} \frac{y}{2}}-1\right)+\frac{h_{4}^{2}}{h_{3}} M_{c 4}\left(e^{h_{3} \frac{y}{2}}-1\right)
\end{array}\right\} \\
& +j \omega b \frac{2 \varepsilon_{33}^{L S}}{\alpha t_{p}}\left(e^{\alpha l}-1\right) .
\end{aligned}
$$

\section{RESULTS AND DISCUSSIONS}

Figure 2 shows the five fabricated actuators $\left(T_{x 1}-T_{x 5}\right)$ whose characteristics of admittance were examined in term of their shape. Among them, $T_{x 1}$ is a conventional rectangular actuator, which is not tapered. The physical properties and dimensions of the actuators are listed in Table 1 . In the calculation, the elas-

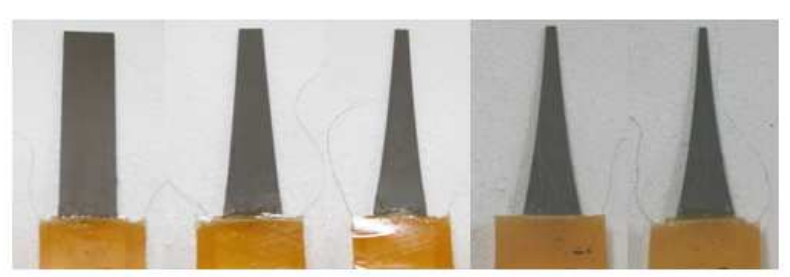

(a) $T_{x 1}(\mathrm{~b}=\mathrm{a})(\mathrm{b}) T_{x 2}(\mathrm{~b}=2 \mathrm{a})(\mathrm{c}) T_{x 3}(\mathrm{~b}=3 \mathrm{a})(\mathrm{d}) T_{x 4}(\mathrm{~b}=4 \mathrm{a})(\mathrm{e}) T_{x 5}(\mathrm{~b}=5 \mathrm{a})$

Figure 2: Photograph of tapered piezoelectric bimorphs.

Table 1: Design factors of bimorph actuator

\begin{tabular}{|r|r|r|r|r|r|}
\hline & $\rho\left(\mathrm{kg} / \mathrm{m}^{3}\right)$ & $\varepsilon_{33}^{T} \times 10^{-11}$ & $k_{32}$ & $b(\mathrm{~mm})$ & \\
\hline & 7370 & 1.6690 & 0.3861 & 12.7 & \\
\hline & $s_{22}^{E} \times 10^{-8}$ & $d_{32} \times 10^{-10}$ & $l(\mathrm{~mm})$ & $t_{p}(\mathrm{~mm})$ & \\
\hline & 3.2669 & 2.8123 & 62.45 & 0.44 & \\
\hline & $T_{x 1}$ & $T_{x 2}$ & $T_{x 3}$ & $T_{x 4}$ & $T_{x 5}$ \\
\hline$a(\mathrm{~mm})$ & 12.7 & 6.3 & 4.2 & 3.2 & 2.0 \\
\hline
\end{tabular}

tic material between the piezoelectric plates is ignored because the adhesive layer $\left(2 t_{m}=0.04 \mathrm{~mm}\right)$ made of epoxy resin is sufficiently thin to ignore and the Young's modulus of the layer is very small compared with that of the piezoelectric plates. All edges of the actuators were machined using a CNC milling machine with on installed CAD/CAM program. The longer of the two linear ends, which were not tapered, was rigidly molded by epoxy to satisfy the fixed-end condition in each actuator. The calculated and measured results of the input free admittance are shown in Figure 3(a) Figure 3(e). In these figures, the solid lines and marks show the theoretical results and measured results, respectively. The results show that the fundamental resonant frequency and each harmonic resonant frequencies are shifted upward with increasing $b / a$. Noted that the fundamental resonant frequency is more sensitive than the harmonic resonant frequencies to the value of $b / a$. The measured and theoretical results are in good agreement, and both of them clearly show the variation of resonant frequency with $b / a$. However, some errors appear in higher harmonics because the elastic material, which was ignored in the theoretical calculation, should not be ignored when the thickness of the plate is comparable to the wavelength of the harmonic mode. Furthermore, the larger the value of $b / a$, the larger the errors eqs. (2a) and ( $2 b)$ are not applicable when the thickness of the bimorph $\left(2 t_{p}\right)$ is comparable to its length $a$. The input admittances are 


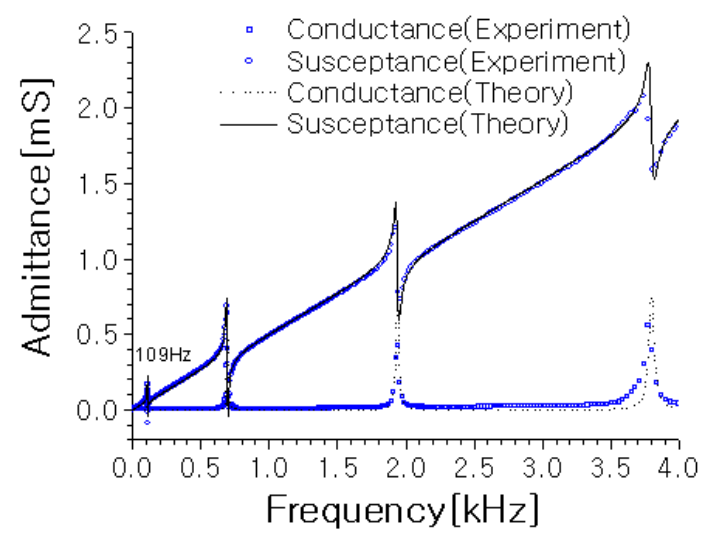

(a) $b=a$

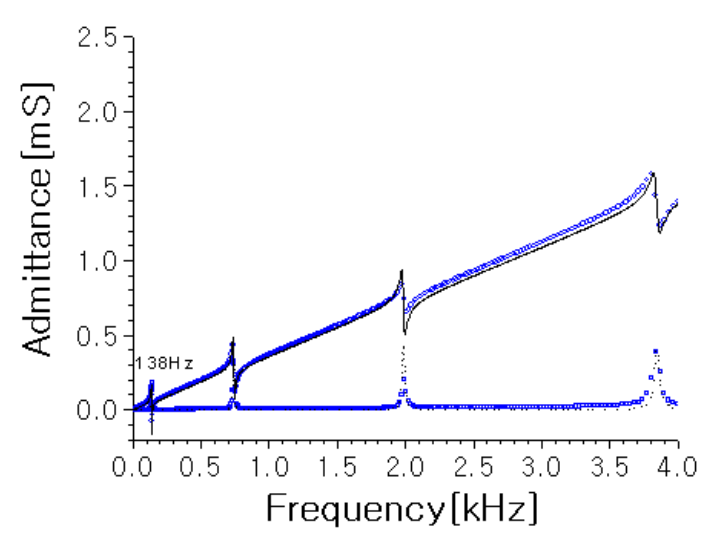

(b) $b=2 a$

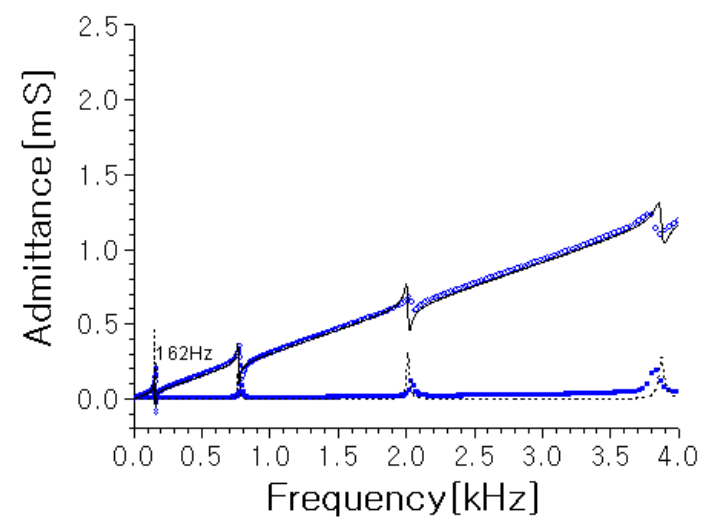

(c) $\mathrm{b}=3 \mathrm{a}$

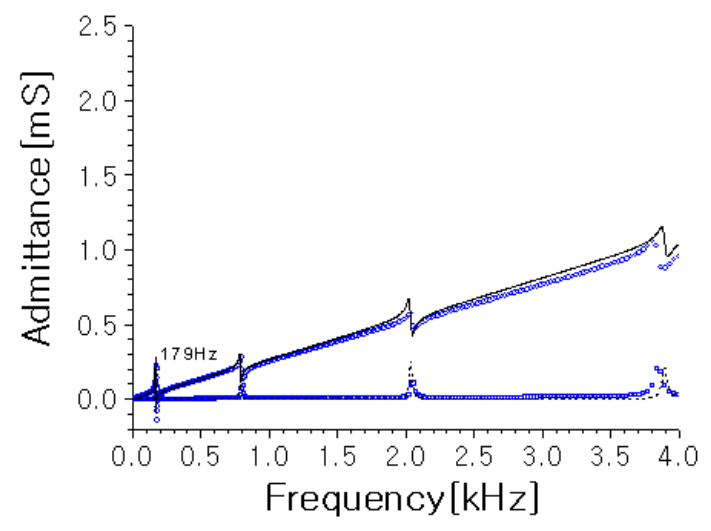

(d) $b=4 a$

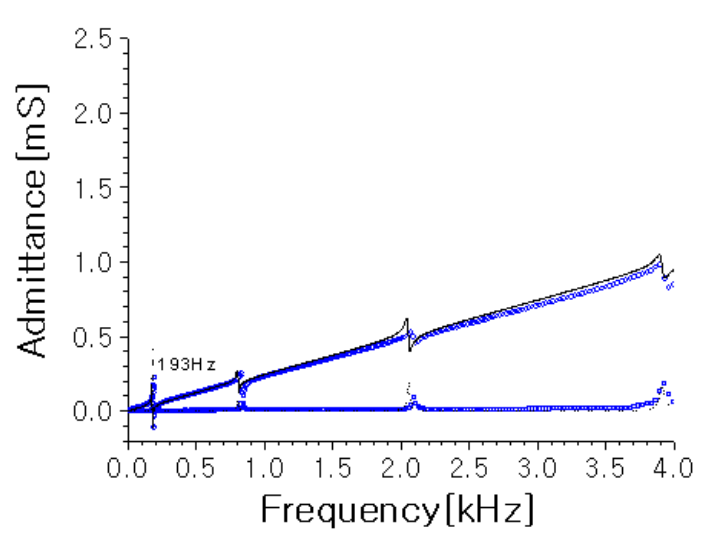

(e) $b=5 a$

Figure 3: Input admittance change with shape.

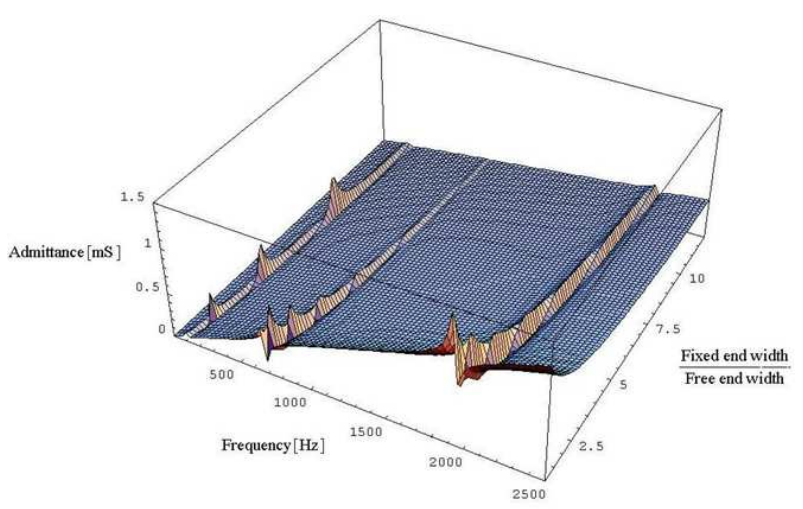

(a)

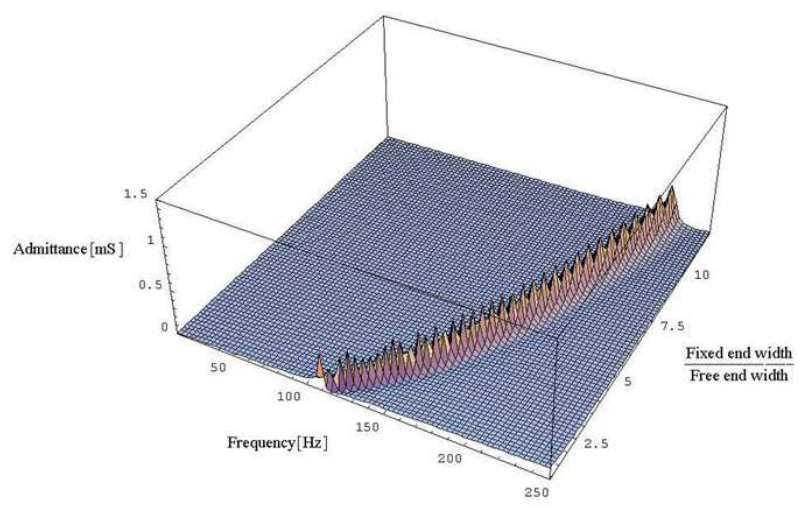

(b)

Figure 4: Change in admittances of the piezoelectric bimorph actuators with frequency and $b / a$. 
calculated for various $b / a$ as shown in Figure 4. Both the admittances and the resonant frequency are significantly changed with the value of $b / a$. Figure 4(b) shows that the fundamental resonant frequency can be controlled from 100 to $230 \mathrm{~Hz}$ in the given range of $b / a$. The peaks in Figure 4(a) are caused by the sparse calculation interval. The calculation results for the fundamental mode obtained with a denser calculation interval are shown in Figure 4(b).

\section{CONCLUSION}

In this study, equations giving the free input admittance and the particle displacement for a piezoelectric bimorph actuator with an exponentially tapered shape were derived. The validity of the equations was confirmed using five fabricated bimorph actuators. The experimental results coincided well with the theoretical results. By analyzing the admittance and displacement using the derived equations, a piezoelectric bimorph actuator with the desired characteristics can be designed. If actuators with various resonant frequencies are arrayed, a thin piezoelectric speaker with a wide bandwidth can be realized.

\section{ACKNOWLEDGMENT}

This work was supported by a Korea Research Foundation Grant funded by the Korean Government (MOEHRD, Basic Research Promotion Fund) (KRF-2008-331-D00222).

\section{REFERENCES}

[1] C. Tanuma, "A Parallel-Bimorph-Type Piezoelectric Actuator for High-Resolution Imager," Japanese journal of applied physics, 38,5603-5607(1999).

[2] H. Tai, J. Seki, T. Kida, and T. Kobayashi, "VibrationType Viscometer Using a Triangular Bimorph Transducer -Measurement of High Viscosity by Low-Frequency Operation," Japanese journal of applied physics, 43, 30883089(2004).

[3] S. J. Kim and J. D. Jones, "Influence of piezo-actuator thickness on the active vibration control of a cantilever beam," Journal of Intelligent Material and Syst. Struct., $6,610-623(1955)$

[4] A. Kruusing, "Analysis and optimization of loaded cantilever beam microactuators," Smart Mater. Struct., 9, 186196(2000)

[5] R. Pedrak, Tzv. Ivanov, K. Ivanova, T. Gotszalk, N. Abedinov, I. W. Rangelow, K. Edinger, E. Tomerov, T. Schenkel, and P. Hudek, "Micromachined atomic force microscopy sensor with integrated piezoresistive sensor and thermal bimorph actuator for high-speed tapping-mode atomic force microscopy phase-imaging in higher eigenmodes," $J$. Vac. Sci. Technol. B, 21, 3102-3107(2003)

[6] J. G. Smits, S. I. Dalke and T. K. Cooney, The constituent equations of piezoelectric bimorph, Sens. Actuators A, 28, 41-61(1991)

[7] J. G. Smits and A. Ballato, "Dynamic admittance matrix of piezoelectric cantilever bimorphs," IEEE. Microelectromech. Syst., 3, 105-111(1994)

[8] T. Iketa, Fundamentals of Piezoelectricity, Oxford Univesity. Press, New York, 1990, pp. 31-60.

[9] Y. Tomikawa, Theory of Ultrasonic Electronic Vibrations, Asakura, Tokyo, 1997, pp. 104-120. 